\title{
Desarrollo y aplicación de software docente en prácticas de psicrometría
}

\author{
Emilio José Sarabia Escriva a , Víctor Soto Francés ${ }^{\mathrm{b}}$ y Jose Manuel Pinazo Ojer ${ }^{c}$ \\ ${ }^{a}$ Universitat Politècnica de València(emsaes@upvnet.upv.es), Universitat Politècnica de \\ València(vsoto@ter.upv.es) y ${ }^{\mathrm{c}}$ Universitat Politècnica de València(jmpinazo@upvnet.upv.es)
}

\begin{abstract}
The article describes the motivation for the development of a friendly software for the students and their integration in the laboratory practices of different subjects related to air conditioning, in which the psychrometric processes are explained.

The software allows to simulate psychrometric processes without having to make costly calculations, and with that, students can better understand the functioning of different equipment.

The text describes the characteristics required for the software in order to improve the learning process and the design of a practice session with it.

Keywords: air conditioning, psychrometry, software

Resumen

El artículo describe la motivación por el desarrollo de un software amigable para los alumnos y su integración en las prácticas de laboratorio de diferentes asignaturas relacionadas con la climatización, en las que se explican los procesos psicrométricos.

El software permite simular procesos psicrométricos sin necesidad de hacer costosos cálculos, con ello, el alumno puede entender mejor el funcionamiento de los diferentes equipos.

En el texto se describen las características requeridas al programa para poder atender a las necesidades docentes y el diseño de una sesión de prácticas con el mismo.
\end{abstract}

Palabras clave: climatización, psicrometría, software

\section{Introducción}

El uso de programas informáticos es una actividad extendida en las sesiones prácticas de muchas asignaturas. Este tipo de actividad permite a los alumnos emplear los conocimientos teóricos vistos en clase en la resolución de casos reales utilizando herramientas que ayudan a realizar los cálculos de forma automática. En muchos casos, el software empleado es de tipo profesional, es decir, no tiene carácter docente. Por ejemplo, en el caso de programas informáticos para selección de equipos, el programa, en base a 
unos datos de entrada te indica el resultado óptimo, sin especificar la metodología de cálculo empleada, los criterios utilizados o las posibles soluciones alternativas. De alguna manera, el programa actúa como una caja negra que responde a unos datos de entrada. Esto puede ser suficiente a nivel profesional, pero a nivel docente esa laguna de información no es adecuada.

En este artículo se describe el desarrollo de un software de carácter docente para la realización de cálculos psicrométricos en asignaturas de climatización. El software también puede emplearse de forma profesional, pero el diseño del mismo ha tenido en cuenta una aplicación docente para su empleo por parte de alumnos en prácticas de climatización.

La psicrometría estudia las propiedades termodinámicas del aire húmedo y el comportamiento de éste ante diferentes procesos como: calentamiento, enfriamiento, humectación, mezcla de diferentes corrientes, etc. De esta forma, se modeliza el comportamiento del aire, con el fin de predecir el estado final del mismo al aplicar uno u otro proceso. Esto requiere conocer las diferentes variables que definen el estado del aire (variables psicrométricas), así como la relación existente entre las mismas (Atecyr, 2009) (Pinazo, 1995).

El estudio de esta materia es un tema básico en aquellas asignaturas relacionadas con la climatización. Existen diferentes grados y másters en la Universitat Politècnica de València (UPV) en los que se imparten asignaturas relacionados con la climatización (Grado de Mecánica, Grado de Energía, Grado de Química, Máster de Tecnologías industriales, Máster de Edificación, Máster de Instalaciones industriales, etc.) por lo que es una asignatura ampliamente extendida.

Los cálculos psicrométricos son necesarios para el diseño de equipos o unidades de tratamiento de aire (UTA). Con ello se diseñan los equipos de clima que son capaces de contrarrestar las cargas térmicas de los edificios (Pinazo, 1996). De ahí la importancia de este tema en cualquier asignatura de climatización. Los cálculos psicrométricos nos permiten, por un lado, determinar las condiciones de impulsión de aire necesarias para compensar las cargas térmicas y por otro, determinar la potencia necesaria que deben tener las baterías de frío y de calor de los sistemas para poder desempeñar este trabajo.

El software implementado está registrado como una patente de software en la oficina del CTT de la UPV con el nombre de SICRO_V2 (código R-18126-2016). En la actualidad, el software es utilizado también por diferentes ingenierías para realizar sus cálculos y se incluye dentro de un paquete de herramientas que ofrece la Asociación de Técnicos de Climatización y Refrigeración para los técnicos del sector de forma gratuita a través de la plataforma (Atecyr, 2016).

El artículo se ha dividido en los apartados: objetivos, en el que se plantea la necesidad de una herramienta docente para la explicación y práctica de conceptos de psicrometría; una sección de desarrollo de la aplicación, en la que se enumeran las características necesarias del software que se desea desarrollar además de su puesta en práctica; una sección de resultados, en la que se comenta cómo ha sido la introducción de la innovación en el aula; y filamente una sección en la que se exponen las conclusiones del trabajo.

(cc) EY-NC-ND 2018, Universitat Politècnica de València 


\section{Objetivos}

El objetivo planteado en el trabajo ha sido desarrollar una herramienta informática de carácter docente para su aplicación en prácticas informáticas de asignaturas de climatización, en concreto para la realización de cálculos psicrométricos. De esta forma, se pueden realizar tareas de diseño de unidades de tratamiento de aire y hacerlo empleando tiempos de cálculo razonables. Además, se preparan una serie de ejercicios, para la sesión de prácticas, que el alumno deberá resolver utilizando la herramienta como apoyo a la hora de realizar cálculos. En el mercado no existe software similar, al menos de carácter docente, por lo que el desarrollo resulta ser innovador en el campo especificado. De hecho, es utilizado en otros centros formativos en que se imparten asignaturas de climatización.

La práctica diseñada se adapta a cualquier asignatura de climatización que contenga el tema de psicrometría. De esta manera, es una tarea que se puede emplear en diferentes asiganturas y cursos, además se puede emplear en cursos presenciales o a distancia. Para las clases de asignaturas presenciales, el profesor hace una explicación básica del funcionamiento de la misma y comenta la actividad que los alumnos deben realizar. En el caso de cursos online, la explicación profesor se substituye por unos videos tutoriales que explican el funcionamiento de la aplicación (Atecyr, 2016). En ambos casos, la entrega de la tarea es un documento con los cálculos realizados en la aplicación y la respuesta a una serie de cuestiones que requieren el análisis de los resultados obtenidos.

\section{Desarrollo de la innovación}

\subsection{Características docentes de la aplicación}

Para el desarrollo de la herramienta se ha utilizado el lenguaje de programación C++ y el compilador de la compañía Borland C++ Builder versión 5 (Schildt ,2001). La herramienta es un ejecutable que funciona en el sistema operativo Windows y el modo de instalación del programa consiste en descomprimir un archivo zip que contiene toda la información del mismo. De esta manera, el programa no requiere ninguna instalación especial y puede ejecutarse incluso desde una unidad USB, esto resulta especialmente útil en aulas informáticas en las que hay que tener permisos especiales para la instalación de software. Esta característica permite también que el alumno pueda tener la aplicación en su propio ordenador y pueda realizar las tareas en cualquier lugar y momento.

A continuación se enumeran aquellas características que hemos considerado interesantes incluir en la herramienta con el fin de conseguir una adecuada función docente:

- Interface sencilla, de manera que el aprendizaje del manejo del programa no suponga una dedicación excesiva del alumno. Para ello el programa contiene únicamente dos pantallas, una principal en la que se definen los procesos psicrométricos y se representan directamente sobre el diagrama psicrométrico y otra en la que se resumen en dos tablas los procesos definidos y las propiedades termodinámicas de las variables en cada punto (Fig. 1). 


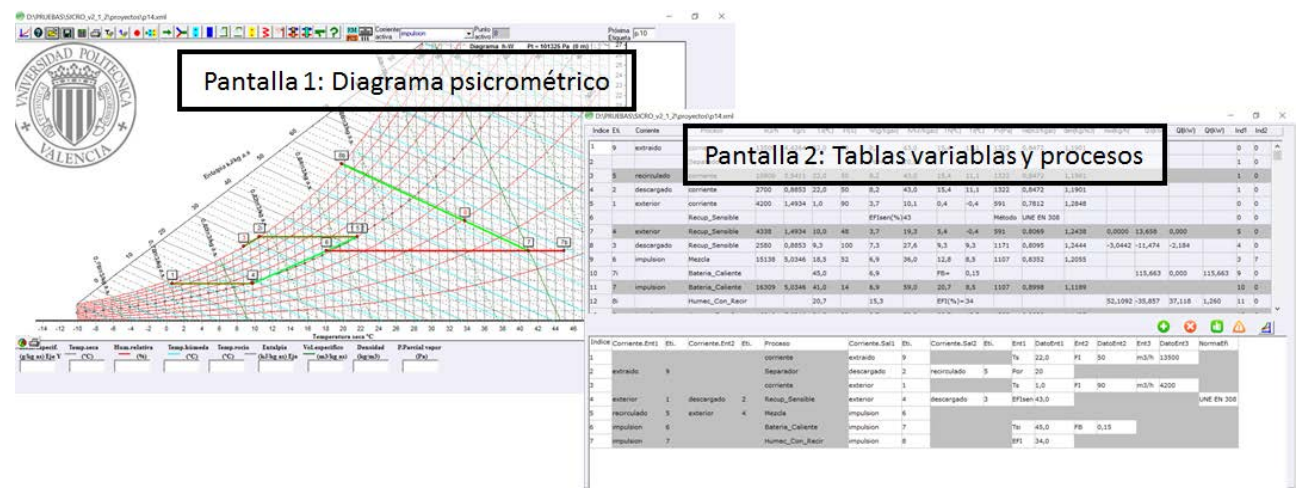

Fig. 1. Pantallas de la aplicación SICRO_V2

- Deglose de los resultados parciales en cada uno de los procesos psicrométricos que se calculan. Esta información permite a los alumnos observar las características de cada uno de los procesos y el efecto que tienen las propiedades de los equipos. Por ejemplo, el paso del aire por una batería caliente proporciona solamente potencia sensible, mientras que el paso por una batería fría puede hacer que exista también una reducción de la humedad del mismo, potencia latente.

- Detalle del valor de todas las variables psicrométricas en cada uno de los puntos de los procesos definidos.

- Posibilidad de modificación de propiedades ya definidas y recálculo del proceso para observar las consecuencias y efectos que producen (Figura 2).

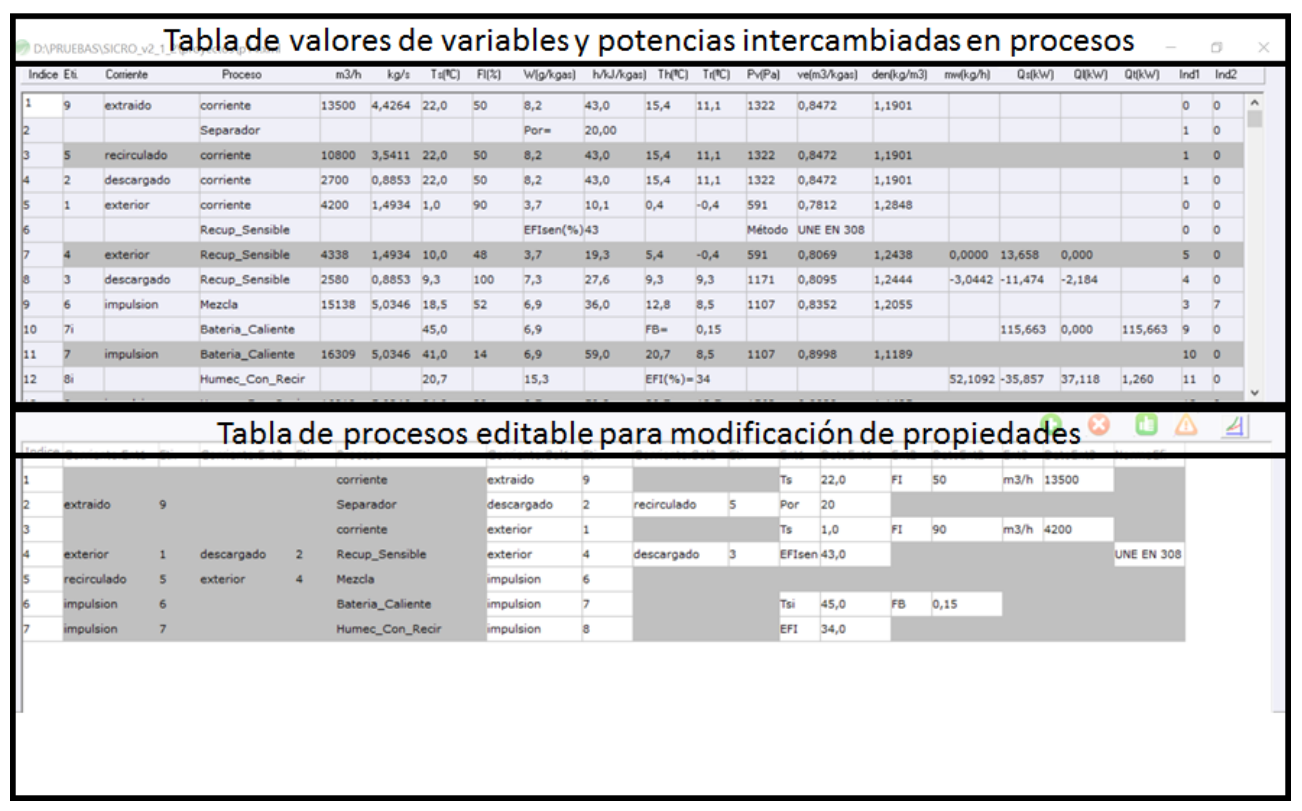

Fig. 2. Tabla de propiedades de los puntos del ciclo y tabla de edición de datos de los procesos.

- Representación gráfica de resultados en el diagrama psicrométrico, con el fin de visualizar de forma clara el proceso general. La representación del proceso sobre

(cc) EY-NC-ND 2018, Universitat Politècnica de València

Congreso IN-RED (2018) 
el diagrama psicrométrico facilita el entendimiento del mismo y de los resultados que se obtienen (Figura 3).

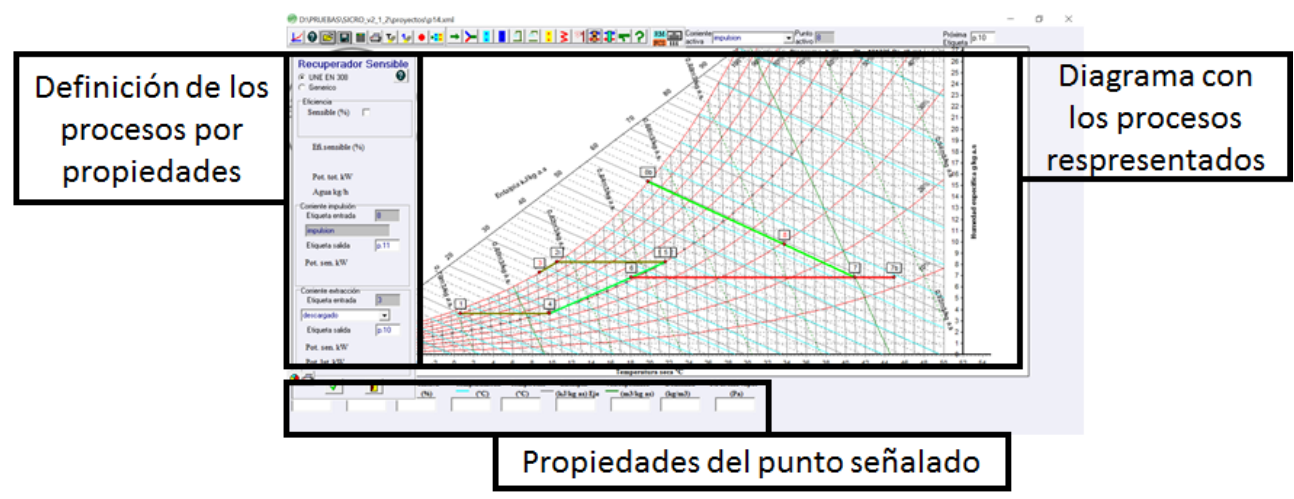

Fig. 3. Diagrama psicrométrico con los procesos representados.

- Informe de resultados de salida en el que se especifiquen los caudales y las propiedades termodinámicas del aire en cada punto. Como formato de salida de resultados se ha decidido emplear html por ser un archivo de texto que se puede visualizar en cualquier editor de texto y navegador.

- Posibilidad de definir los procesos psicrométricos de diferentes formas, incluso de modo gráfico.

- Creación de tutoriales explicativos del manejo de la instalación para que los alumnos presenciales puedan volver a ver algunas características del programa y para que los alumnos de cursos a distancia puedan aprender el manejo de la aplicación. Los tutoriales consisten en videos cortos de entre 5 y diez minutos en los que se explican detalles concretos del programa (Atecyr, 2016).

- Versión del programa en inglés para poder utilizarla con alumnos extranjeros o con los grupos asignados a esta lengua.

\subsection{Diseño de una práctica informática con la aplicación}

A continuación se describe una sesión práctica de tres horas de duración dedicada al análisis del funcionamiento de dos UTAs (unidades de tratamiento de aire) utilizando el programa SICRO_V2 para la resolución y discusión de resultados. La práctica se ha diseñado a modo de análisis de dos casos de equipos de climatización. Una situación que el alumno debería entender como motivadora, lo cual ayuda a la implicación del alumno en la resolución de la misma y facilita el aprendizaje. (Cabrero, 2006) especifican las variables que deben tenerse en cuenta para que la actividad sea percibida como útil por parte de los alumnos.

El esquema de la sesión práctica es sencillo, una primera hora se dedica a la explicación del manejo del programa por parte del profesor y con ello se repasan algunos conceptos ya vistos en las sesiones teóricas. En el caso de los cursos online, esta hora está dedicada por 
parte del alumno a la visualización de los videos tutoriales de la aplicación que muestran el funcionamiento de la misma (Atecyr, 2016).

Las siguientes dos horas están dedicadas al trabajo individual del alumno para la resolución de dos modos de funcionamiento que se plantean para una equipo de climatización. Cada uno de los ejercicios tiene una duración de una hora. La tabla 1 refleja el ajuste de tiempos asignados a cada actividad.

Tabla 1. Esquema de práctica informática con el programa SICRO

\begin{tabular}{cc}
\hline Tareas & Duración \\
\hline $\begin{array}{c}\text { Caso presencial: Explicación del } \\
\text { funcionamiento del programa. }\end{array}$ & 1 hora \\
Caso online: Visualización videos \\
tutoriales del manjo de la aplicación \\
$\begin{array}{c}\text { Tarea 1. Análisis funcionamiento de } \\
\text { una UTA en modo verano }\end{array}$ \\
$\begin{array}{c}\text { Tarea 2. Análisis funcionamiento de } \\
\text { una UTA en modo invierno }\end{array}$ \\
\hline
\end{tabular}

Las tareas 1 y 2 consisten en el análisis del funcionamiento de unos equipos de climatización, uno para modo verano y otro para modo invierno. La figura 4 muestra el esquema de estas UTAs que el alumno deberá definir en la aplicación SICRO_V2 y responder a una serie de cuestiones para identificar que entiende los resultados que la aplicación muestra y que es capaz de utilizarlos para realizar otros análisis.
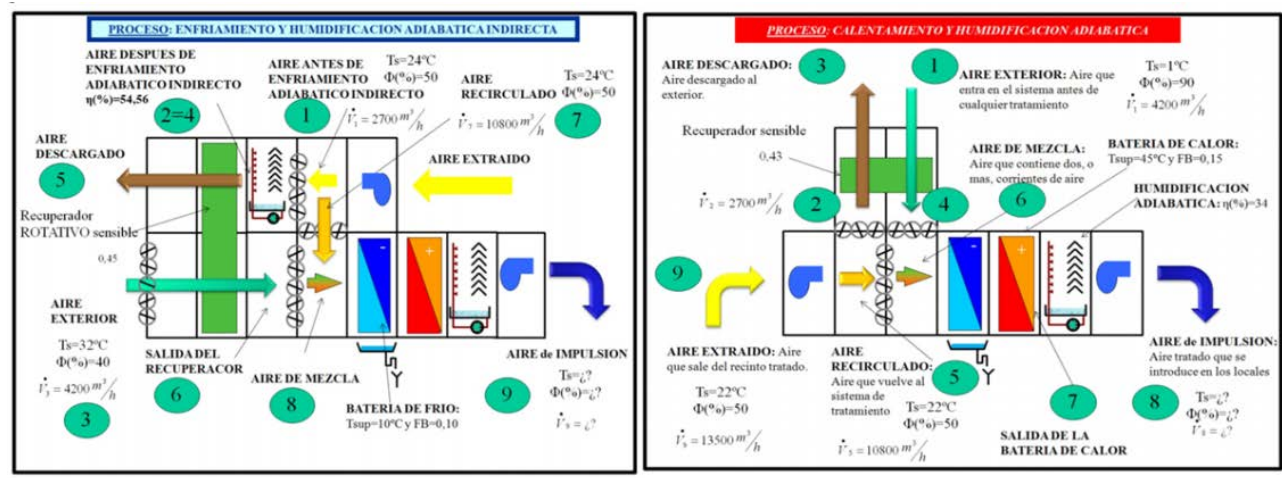

Fig. 4. Equipos de climatización cuyo comportamiento el alumno debe modelizar en la aplicación.

El alumno deberá definir cada uno de los procesos psicrométricos que existen en las diferentes corrientes de aire definidas en las figuras para responder a las siguientes cuestiones:

- Cuestiones conceptuales: 
o Conocer las propiedades termodinámicas de cada uno de los puntos indicados en los equipos.

o Determinar la potencia intercambiada con el aire por cada uno de los equipos.

- Cuestiones de análisis:

o Indicar el significado de los valores positivos y negativos en las potencias.

o Calcular las cargas térmicas que pueden contrarrestar estos equipos en un edificio.

o Indicar qué consecuencias tiene si no existe humectador en el aire de extracción para la UTA que trabaja en modo verano.

o Indicar qué pasa cuando el recuperador sensible tiene una eficiencia del $60 \%$ en ambos casos.

Las primeras dos cuestiones se resuelven identificando los datos correctos sobre los resultados de la aplicación, son cuestiones conceptuales. Mientras que para el resto de cuestiones, el alumno hace uso de los resultados obtenidos para llegar a otras conclusiones, se trata de cuestiones de análisis.

La evaluación de la práctica se especifica en la Tabla 2. A modo de resumen, se valora en un $30 \%$ el diseño de los equipos en el programa SICRO_V2 y un 30\% conocer dónde identificar las variables del proceso y los resultados obtenidos. El 40\% restante corresponde a la resolución de las cuestiones de análisis propuestas.

Tabla 2. Evaluación de la práctica

\begin{tabular}{ccc}
\hline Tareas & Actividad & Valor \\
\hline \multirow{2}{*}{$\begin{array}{c}\text { Tarea 1. Análisis funcionamiento de } \\
\text { una UTA en modo verano }\end{array}$} & Diseño de la UTA & $15 \%$ \\
\cline { 2 - 3 } & Cuestiones conceptuales & $15 \%$ \\
\hline \multirow{2}{*}{$\begin{array}{c}\text { Tarea 2. Análisis funcionamiento de } \\
\text { una UTA en modo invierno }\end{array}$} & Cuestiones de análisis & $20 \%$ \\
\cline { 2 - 3 } & Cuseño de la UTA & $15 \%$ \\
\cline { 2 - 3 }
\end{tabular}

\section{Resultados}

El uso del programa informático SICRO_V2 ha permitido desarrollar una práctica exclusivamente informática sobre la materia. Este tema se complementa en las asignaturas de clima con otra sesión de prácticas en el laboratorio, sobre equipos reales. Las sesiones de laboratorio con equipos reales permiten que el alumno pueda ver in situ cómo es y funciona una instalación real, lo cual les motiva y mejora el proceso de aprendizaje. Pero esta sesión práctica en el laboratorio se aprovecha mucho más cuando el alumno llega con los conceptos teóricos claros, si no es así, el profesor debe invertir mucho tiempo en repasar 
parte del contenido teórico para que los alumnos entiendan adecuadamente lo que está pasando en los equipos.

Se ha observado que la sesión de laboratorio se aprovecha más cuando la sesión informática se realiza previamente, ya que ésta le permite al alumno entender mejor cada uno de los procesos psicrométricos y el funcionamiento de los equipos. El programa informático permite modificar el valor de algunas propiedades de equipos y ver qué consecuencias tiene eso sobre el proceso final. En el laboratorio, en cambio, los equipos tienen menor posibilidad de variabilidad y cuando se realizan cambios, los resultados no son tan inmediatos ni fáciles de entender. Esto se comprueba al analizar los diferentes trabajos de prácticas presentados por los alumnos. Aquellos grupos que han trabajado la sesión informática previamente son capaces de entender mejor el comportamiento de los equipos del laboratorio cuando obtienen los datos.

Por tanto, la sesión informática con el programa SICRO_V2 permite a los alumnos entender mejor los procesos psicrométricos y con ello el funcionamiento de los equipos de climatización. Por otro lado, se considera necesario también para el correcto aprendizaje de la materia una sesión de laboratorio en la que los alumnos pueden visualizar los equipos y tomar ellos los datos para la realización de cálculos.

\section{Conclusiones}

Para concluir enumeramos algunos de los aspectos más significativos del trabajo que se ha realizado:

- Se ha desarrollado una aplicación informática de carácter docente para las asignaturas de climatización. En concreto para el tema de psicrometría.

- La herramienta cumple con las especificaciones que se han indicado en el apartado de desarrollo y que se han considerado interesantes para un trabajo adecuado con los alumnos.

- Se ha diseñado una sesión de prácticas informáticas de una duración de tres horas que puede ser impartida tanto en clases presenciales como en cursos online.

- El uso de la herramienta no tiene por qué limitarse a la sesión de prácticas, también se puede utilizar en las sesiones de teoría y de problemas de las asignaturas con el fin de ilustrar mejor las explicaciones.

- Cuando los alumnos trabajan primero la sesión informática entienden mejor el comportamiento de los equipos en las sesiones de laboratorio, como se comprueba al comparar los trabajos de prácticas.

\section{Referencias}

ATECYR (2009), Psicrometría DTIE 3.01. Madrid, Atecyr

ATECYR (2016). Calcula con Atecyr. <http://www.calculaconatecyr.com/> [Consulta: 16 de mayo de 2018]

(cc) EY-NC-ND 2018, Universitat Politècnica de València

Congreso IN-RED (2018) 
ATECYR (2016). Tutorial SICRO_V2. http://www.calculaconatecyr.com/tutoriales-psicro.php [Consulta: 16 de mayo de 2018]

CABRERO, J., ROMAN, P (2006). E-actividades: un referente básico para la formación en internet. Editorial MAD.

PINAZO, JM (1995) Manual de climatización. Tomo I:Transformaciones sicrométricas. Editorial UPV

PINAZO, JM (1996) Manual de climatización.Tomo II:Cargas Térmicas. Editorial UPV

SCHILDT, H., GUNTLE, GL (2001) Borland C++ Builder: The Complete Reference. Editorial Osborne/McGraw-Hill 